\title{
Sexual dysfunctions in the spinal cord injured
}

\author{
R Stien \\ Laboratory of Neurourology, Dept of Neurology, Rikshospitalet, Oslo, Norway.
}

The function of the human sexual organs and the ability to experience pleasure from physical intimacy are dependent on a well functioning and integrated peripheral and central nervous system. Afferent influx from the genital organs, skin, auditory, visual and gustatory stimuli and phantasies are conveyed to central, para-midline and temporal areas of the brain. The control of sexual behaviour and the conscious experience of erotic pleasure and orgasm are located in these centres. The only difference between SCI patients and others is the disturbed connection between the lower part of the body including the genital organs, and the higher centres of the brain. As our personality is not located in the legs, and as our main sexual organs are located between the ears, not between the legs, the SCI patient has the same sexual desires as any other person. It is further important to realize that human sexual behaviour is not necessarily an escalating series of acts leading from gentle touch via petting to intercourse and ejaculation. All kinds of physical intimacy with all kinds of outcome may be as satisfactory. It should be pointed out that 'de-genitalised' sexual behaviour may be mutually acceptable and pleasurable. On the other hand, if a SCI man feels that erections and vaginal penetration is the ultimate goal of his sexualilty, it is an inappropriate therapeutic approach to try to persuade him that this is not necessary. As therapists we shall try to help, not to impose our personal views onto the patient.

\section{Desire (libido) dysfunctions}

SCI patients may have libido dysfunctions even if, as stated above, they should have the same sexual desires as other people. One reason might be that they live in societies that try to 'de-sexualise' certain groups like the elderly and the physically handicapped. Luckily, in the last part of this century attitudes have changed dramatically in many countries. From sexual suppression we have turned to a debate on whether handicapped people have a right to sexual life or even should be helped by professional sex therapists to some kind of sexual satisfaction. ${ }^{1}$

Another main origin of lack of sexual desire is the patient's disturbed body image. Patients should be helped to reject the idea that they are not sexual persons and are unattractive. Open discussions with other SCI patients are often the best treatment. These discussions should be started early after injury. Discussions must be directed by someone with a relaxed relationship to his/her own sexual life, and embarrassed and red faced therapists should be replaced immediately. Important parts of the restoration of self esteem and body image are maximal physical rehabilitation, treatment of urinary problems (no permanent catheters) and avoidance of sores.

Heavy medication and sedation may destroy sexual desire, but untreated pain or spasms may have the same adverse effect.

\section{Problems with orgasm}

Orgasm, the ultimate sexual climax of pleasure, is a psychological experience depending on intact function of 'sexual centres' in the brain. It is important to realise that it is possible to have orgasms without any motor or sensory function in the genital organs including the pelvic floor muscles. Furthermore, no 'partner will 'give' the other an orgasm. It is one's own responsibility to act and guide the partner in such a way that one reaches a climax.

Female SCI patients are easier to guide and treat in this respect than males. When there is little or no sensory function in the genital region, it is important to investigate 
the erotic possibilities of the rest of the body: the ears, neck, nipples, back etc. By careful exploration and use of these possibilities the female SCI may have orgasms as delightful as they were before the injury. These orgasms are often depicted as being 'phantom' or 'mental', but that is to classify them as being not normal, which tells us more about the attitudes of the describer than the quality of the orgasm.

Women are superior to men in other ways: they may have serial orgasms, which in my experience never occur in SCI men and hardly in normals. Men are traditionally, and perhaps biologically, more dependent on sensory function in the genitals to obtain an orgasm. Usually SCI men with complete lesions will not climax, but the same technique as is described for women may be successful. Prolonged genital stimulation may bring the patient to a climax, with or without an ejaculation. It should be stressed that ejaculation is not an integral part of orgasm.

Finally, the hunt for orgasms should not be given too high a priority. Again women are the easiest to guide: an intimate relationship may be quite satisfactory without orgasms. Sexuality is far more than achieving an orgasm, and any kind of physical intimacy, with any person of any sex may be quite acceptable and pleasureable without orgasms.

\section{Problems with erection and lubrication}

Male erection and lubrication in females are controlled by the same neurophysiological mechanisms. Basically it is a neurogenic control of arterioles (increased bloodflow) and venous structures (decreased drainage) in the genitals. Both sympathetic (lower thoracic level) and parasympathetic (sacral level) nerves take part in this control. The sacral medulla is intact in most SCI patients, and erection and lubrication can usually be achieved by local stimulation of the genital region, by bladder filling or by spasms. Psychogenic erections may be normal in patients with lumbar lesions, leaving the connections with lower thoracic levels intact. About $80 \%$ of SCI men will experience some erectile ability. The overwhelming problem is the duration of the erection: tumescence and rigidity tend to fade away rather quickly leaving the patient unable to perform intercourse. ${ }^{2}$

Difficulties with lubrication are easily dealt with by prescribing an inert 'personal lubricant'. These lubricants should rather be on the acid side of the $\mathrm{pH}$-scale so as not to disturb the natural vaginal environment. When adductor or pelvic floor spasticity makes penetration difficult, the use of benzodiazepines as best antispastic adjuvant is advocated.

The symptomatic treatment of erectile dysfunction can be described with reference to 3 aids:-

1 Technical aids. Most SCI men can produce a good erection that disappears too quickly. A tight ring placed around the base of the penis will block the venous drainage. A penis ring should be made of a good quality elastic material, should be very tight ( 2 rings), easy to put on (loader devices exist) and easy to take off ('ears' on the tension rings). If the patient has no erectile ability, the tension ring can be supplied by the use of a vacuum pump. A supporting condom may be another solution to his problem.

2 Pharmacological erection. There are 2 groups of drugs that taken orally may improve erection: alpha-adrenergic blocking agents (yohimbine) and dopamine agonists (levodopa). None are effective in SCI men in my experience. Intracavernous self injections with alpha-adrenergic blocking agents (phenoxybenzamine, papaverine-alone or mixed with phento lamine), prostaglandins $\left(\mathrm{PGE}_{1}\right)$ or various neuropeptides (VIP) are very effective. The technique is easily learned by the patient or partner. The pros and cons for the different drugs are: alphablockers (inexpensive, high rate of priapism, local fibrosis); prostaglandins (no priapism, local pain, relatively expensive); neuropeptides (resembles 'normal' erection, expensive). New and better drugs will be found and tried, and better injection systems (like insulin-pens) will be deve- 
loped. Cutaneous application of drugs is also possible. ${ }^{3}$

3 Implantation of a penile prosthesis. There are different implant systems: semirigid, 'string' types and inflatable systems. ${ }^{1}$ We nearly always use semirigid implants. Patients are very carefully selected; post surgery infection or rejection of the implant can be troublesome in SCI patients. All other kinds of treatment must be tried first. Extra indications are age (middle age, elderly); concomitant vascular erectile dysfunction; or the need for better conditions for fastening a uridome. When reviewing 50 of our patients on the question: 'What is your main complaint?' a surprisingly common answer was: 'I was told about the implant too late!'

\section{Ejaculatory dysfunction}

The ejaculation reflex is dependent on intact spinal levels from the lower thoracic (sympathetic: emission of sperm into urethra, closure of bladder neck) to lumbar/sacral (afferents from genitals, somatic efferent innervation of ejaculatory muscles). Centres in the brain (hypothalamus) control the reflex. Less than $10 \%$ of males with complete SCI lesions will experience some kind of semen emission, retrograde or anterograde ejaculation. Drugs may have an adverse effect on ejaculation (alcohol, antidepressants, alphablockers, benzodiazepines). Ejaculatory dysfunction is only a problem when fertility is considered. For the time being 3 methods of artificial ejaculation are available:

1 Intrathecal drug injection. Choline-esterase inhibitors (neostigmine) may induce single or repeated ejaculations in $60-70 \%$ of SCI men when injected intrathecally. Serious side effects (rise in blood pressure, intracranial haemorrhages) have caused this method to be little used even when the injection of prostaglandins $E_{2}$ can block the rise in blood pressure. ${ }^{4}$ Subcutaneous injection of physostigmine combined with butylhyoscine (to prevent peripheral cholinergic effects) and metoclopramide (to pre- vent vomiting) may be used to facilitate reflex ejaculation brought about by the techniques described below. ${ }^{5}$

2 Electroejaculation. Electrical stimuli applied to the rectal mucosa over the seminal vesicles or even to the bulbocarvernous muscles or the skin in the same region may elicit an ejaculation. Solid rectal electrodes ${ }^{6}$ or finger mounted electrodes seem to be most effective. Seventy to eighty per cent of SCI men will produce an ejaculation with this method. Fairly high electrical voltage or strong currents may be needed, and the risk of autonomous dysreflexia, heart arrythmias and mucosal burns must be taken into consideration.

3 Vibroejaculation. The application of strong vibration $(80 \mathrm{~Hz})$ to the frenulum area or shaft of the penis will provoke an ejaculation in about $60-70 \%$ of anejaculatory SCI men. Vibroejaculation in patients with high lesions (above T5) may give autonomous dysreflexia. Vibration is least successful in lesions below T8. In order to improve semen quality or perform insemination, couples may try vibroejaculation at home. Small, inexpensive vibrators with large amplitude (3-4 $\mathrm{mm})$ are then needed.

Hypergastric plexus stimulators may be a solution for those who cannot obtain an ejaculation by other methods. Implanted capsules connected to the vas deferens have so far been of little success even if it is possible to withdraw living spermatozoa from them. ${ }^{7}$

\section{Infertility}

Female SCI patients have no large hormonal or menstrual dysfunction. Their fertility is only slightly decreased, partly due to psychogenic reasons. They have fear of complications during pregnancy and worries about child raising whilst being wheelchairbound. Pregnancy and delivery may be complicated by rise in blood pressure and heart arrythmias. We recommend pregnancy control and delivery in close cooperation between the gynaecologist and an 
experienced SCI specialist. In our clinic, patients are referred to hospital 2-4 weeks before expected delivery and Caesarean section is performed within the last 2 to 3 weeks. Other centres have as good results with normal deliveries at full term.

SCI men tend to have low sperm quality. ${ }^{8}$ There is usually a high number of sperm cells with abnormal morphology and low motility. After serial ejaculations the quality will improve, the percentage of motile spermatozoa being the most valid parameter. Still the pregnancy rate is lower when inseminating with sperm from SCI men compared with anonymous normal donors (one in 10 trials as compared to one in 2). The reason for this is not well known: a raised scrotal temperature may partly explain this, but cooling does not seem to solve the problem. Altered sperm fluid is not the full explanation, as sperm from SCI men and fluid from donors does not change the quality.

Vibration seems to yield semen of better quality than electroejaculation. Judged by the fructose level of ejaculates, vibroejaculation give a high proportion of 'young' sperm cells from the bi-testicles. On the other hand, some 'loading' with energy (fructose) in the seminal vesicles is also considered to be beneficial to the spermatozoa.

The quality of semen is not affected by the level of the lesion or the time from the day of the injury. In our series we have a man now aged 37, who was injured when 11 years old (complete T6), and had never ejaculated until we started vibroejaculation 22 years after the injury. After 5 vibroejaculations his wife was inseminated, with positive results. They are now expecting their second child. The mode of insemination may influence the success rate: insemination at home may be better than at hospital. Twenty-five to thirty per cent of our pregnancies have been achieved in this way. Hormonal stimulation of the female partner may help the timing of the ovulation and insemination.

Techniques where the most viable sperm cells are collected ('swim up') combined with in vitro fertilization have been very promising (success in 2 out of 3 attempts).

Further research into the reasons for low sperm quality in SCI men will undoubtedly provide better techniques for improvements. This fact, combined with the possibility of performing in vitro fertilization on a large scale, will probably solve most of the infertility problems of SCI men.

\section{References}

1 Leyson JFJ (ed) (1991) Sexual Rabilitation of the Spinal Cord Injured. Humana Press, Clifton, New Jersey.

2 Comarr AE (1970) Sexual function among patients with spinal cord injury. Urol Int 23: 134-168.

3 Lue TF, Tanagho EA (1987) Physiology of erection and pharmocological management of impotence. J. Urol 137: 829-836.

4 Guttmann L. Walsh JJ (1971) Prostigmin assessment of fertility in spinal man. Paraplegia 1: 39-50.

5 Chapelle PA, Blanquart F, Puech AJ, Held JP (1983) Treatment of anejaculation in the total paraplegic by subcutaneous injection of physostigmine. Paraplegia 21: 30-36.

6 Halstead LS, VerVoort S, Seager, SWJ (1987) Rectal probe electro-stimulation in the treatment of anejaculatory spinal cord injured men. Paraplegia 25: 120-129.

7 Brindley GS (1986) Sexual and reproductive problems of paraplegic men. In: Clarke JR, ed. Oxford Reviews of Reproductive Biology, 8. Clarendon Press, Oxford: 214-222.

8 Brindley GS (1984) The fertility of men with spinal injuries. Paraplegia 22: 337-348. 